\title{
Cognitive Function and 3-Tesla Magnetic Resonance Imaging Tractography of White Matter Hyperintensities in Elderly Persons
}

\author{
William Reginold ${ }^{a} \quad$ Angela C. Luedke ${ }^{b} \quad$ Angela Tam $^{b} \quad$ Justine Itorralba ${ }^{b}$ \\ Juan Fernandez-Ruiz ${ }^{b}$ Jennifer Reginold ${ }^{d}$ Omar Islam $^{c}$ \\ Angeles Garcia ${ }^{a, b}$ \\ ${ }^{a}$ Memory Clinics, Division of Geriatric Medicine, Department of Medicine, ${ }^{b}$ Centre for \\ Neuroscience Studies, and 'Department of Diagnostic Radiology, Kingston General \\ Hospital, Queen's University, Kingston, Ont., and ' Life Sciences, University of Toronto, \\ Toronto, Ont., Canada
}

\section{Key Words}

White matter hyperintensities · Diffusion tensor imaging · Tractography · Cognitive function

\begin{abstract}
Background/Aims: This study used 3-Tesla magnetic resonance imaging (MRI) tractography to determine if there was an association between tracts crossing white matter hyperintensities $(\mathrm{WMH})$ and cognitive function in elderly persons. Methods: Brain T2-weighted fluid-attenuated inversion recovery (FLAIR) and diffusion tensor MRI scans were acquired in participants above the age of 60 years. Twenty-six persons had WMH identified on T2 FLAIR scans. They completed a battery of neuropsychological tests and were classified as normal controls $(n=15)$ or with Alzheimer's dementia $(n=11)$. Tractography was generated by the Fiber Assignment by Continuous Tracking method. All tracts that crossed WMH were segmented. The average fractional anisotropy and average mean diffusivity of these tracts were quantified. We studied the association between cognitive test scores with the average mean diffusivity and average fractional anisotropy of tracts while controlling for age, total $\mathrm{WMH}$ volume and diagnosis. Results: An increased mean diffusivity of tracts crossing WMH was associated with worse performance on the Wechsler Memory Scale-III Longest Span Forward $(p=0.02)$. There was no association between the fractional anisotropy of tracts and performance on cognitive testing. Conclusion: The mean diffusivity of tracts crossing WMH measured by tractography is a novel correlate of performance on the Wechsler Memory Scale-III Longest Span Forward in elderly persons.


Reginold et al.: Cognitive Function and 3-Tesla Magnetic Resonance Imaging

Tractography of White Matter Hyperintensities in Elderly Persons

\section{Introduction}

White matter hyperintensities (WMH) are high-signal lesions visible on T2-weighted magnetic resonance imaging (MRI) scans that are representative of cerebral small-vessel disease and have been associated with cognitive decline and dementia [1]. WMH were found in $21 \%$ of individuals around the age of 64 years and $94 \%$ of individuals around the age of 82 years [2]. It has been recommended that physicians consider cognitive screening in patients with incidental WMH [3]. Based on these recommendations, the majority of the elderly population would receive cognitive screening. There is a need for MRI measures that can, out of this large group of elderly people with $\mathrm{WMH}$, identify patients at higher risk of poor cognitive function that may benefit from cognitive screening. There is currently no macrostructural or microstructural feature of WMH that reliably associates with cognitive function. The association between WMH lesion volumes and cognitive function is inconsistent and weak [4, 5]. Diffusion tensor imaging (DTI) has also been used to measure the microstructural integrity of WMH. Reduced mean diffusivity (MD) and increased fractional anisotropy (FA) were found in the WMH themselves [6], but these DTI measures correlated with cognitive function in some studies [7, 8] but not in others [9-11]. Tractography is a recent methodological advancement in the application of DTI that may help better relate WMH to cognitive function.

According to the 'disconnection' hypothesis, cognitive decline is associated with the disruption of white mater tracts [12]. The integrity of 'disconnected' tracts may correlate better with cognitive function than the integrity of the lesion itself. In DTI tractography, fiber tracts are traced in three-dimensional space, and the MD and FA of the tracts can be assessed [13]. Tractography has been used to correlate the integrity of white matter tracts with cognitive function in healthy elderly persons [14-20] and patients with dementia [21-24]. Tractography studies of ischemic infarcts and multiple sclerosis plaques have found correlations between the integrity of affected tracts and cognitive function [25]. Earlier tractography studies of WMH used the tract-based spatial statistics (TBSS) procedure [26-28]. In these earlier TBSS studies, each subject's FA map was combined to generate a group mean FA skeleton representing all tracts common to subjects with WMH, and the MD/FA of common tracts were measured [26]. These studies detected associations between the MD/FA of common tracts and performance on several cognitive tests including the MMSE [28], Trails, Stroop test [26], Wisconsin Card Sorting, Longest Span Forward and Longest Span Backward [27]. The studies concluded that, in subjects with WMH, the MD/ FA of white matter tracts were related to cognitive function and found this supportive of the 'disconnection' hypothesis. These white matter tracts may or may not have crossed WMH in each subject. Since measurements were from a mean FA skeleton for the entire study group, the TBSS procedure prevented earlier studies from specifically measuring tracts crossing WMH, which would have varied between subjects. For this study, we used deterministic tractography by the Fiber Assignment by Continuous Tracking (FACT) procedure to create unique fiber tracts for each subject. By measuring only tracts that crossed WMH in each subjects (WMH tracts), we could better assess the 'disconnection' hypothesis. In a cohort of elderly persons with WMH, we studied the association between cognitive test scores (see below) and WMH tract FA and MD while controlling for age, dementia diagnosis and volume of WMH. If tractography of WMH correlated with cognitive function, it would have the potential to help triaging patients with incidental WMH for cognitive screening. 
Table 1. Demographic and clinical data for participants with and without Alzheimer's dementia

\begin{tabular}{lccc}
\hline & Total & Cognitively normal & Alzheimer's dementia \\
\hline Subjects, $\mathrm{n}$ & 26 & 15 & 11 \\
Age, years & $73.8 \pm 8.3$ & $72.5 \pm 9.3$ & $75.7 \pm 6.5$ \\
Male/female gender, $\mathrm{n}$ & $9 / 17$ & $4 / 11$ & $5 / 6$ \\
WMH volume, voxels & $2,618.3 \pm 5,047.1$ & $1,653.2 \pm 3,793.5$ & $3,934.5 \pm 6,342.9$ \\
MMSE score & $26.9 \pm 2.8$ & $29.0 \pm 1.3$ & $24.0 \pm 1.4$ \\
Longest Span Backward & $7.2 \pm 1.7$ & $8.1 \pm 1.2$ & $6.1 \pm 1.6$ \\
Longest Span Forward & $7.6 \pm 1.6$ & $8.3 \pm 1.2$ & $6.5 \pm 1.4$ \\
Stroop test & $74.1 \pm 23.8$ & $89.0 \pm 13.6$ & $53.8 \pm 19.3$ \\
Letter Number Sequencing & $9.5 \pm 3.3$ & $11.3 \pm 2.3$ & $7.1 \pm 2.9$ \\
\hline
\end{tabular}

Values are means \pm standard deviations unless otherwise stated.

\section{Methods}

\section{Participants}

The study was approved by the research ethics board of Queen's University. All participants provided written informed consent prior to entering the study. The neuropsychological battery consisted of the following cognitive tests: MMSE [29], Wechsler Memory Scale-III Longest Span Backward [30], Wechsler Memory Scale-III Longest Span Forward [30], Stroop test [31] and Letter Number Sequencing [30]. The clinic director (A.G.) diagnosed Alzheimer's dementia following the NINCDS-ADRDA criteria [32]. There were no participants with mild cognitive impairment included in this study. All participants with Alzheimer's dementia were in the mild stages of the disease. Exclusion criteria included persons younger than 60 years of age, the presence of metallic objects, devices or conditions unsafe for the MRI, or if they did not have WMH in T2 FLAIR as assessed by a board-certified neuroradiologist (O.I.). All participants had a 3-Tesla MRI brain scan within 2 weeks of cognitive testing. A total of 91 participants had MRI scans, of which 26 subjects met inclusion criteria for the study (presence of WMH and age 60 years or over). The cohort included 15 persons without dementia and 11 persons with Alzheimer's dementia, allowing for a wide range of cognitive function and scores on psychometric tests. The demographic characteristics of the participants are shown in table 1.

\section{Magnetic Resonance Imaging}

All brain imaging was acquired in one session on a 3-Tesla Siemens Magnetom Trio MRI system (Siemens Medical Systems, Erlangen, Germany) with the use of a 12-channel head coil. A high-resolution anatomical scan was acquired with a sagittal T1-weighted three-dimensional magnetization prepared rapid gradient echo (MP-RAGE) sequence [field of view (FoV) $256 \mathrm{~mm}$, spatial resolution $1 \times 1 \times 1 \mathrm{~mm}^{3}$, repetition time (TR) 1,760 ms, echo time (TE) 2.2 ms, flip angle $9^{\circ}$, number of slices 176]. An axial T2-weighted two-dimensional fluid-attenuated inversion recovery (FLAIR) sequence interleaved scan was acquired for detecting WMH (FoV $250 \mathrm{~mm}$, voxel size $1 \times 1 \times 3 \mathrm{~mm}^{3}$, TR 9,000 ms, TE $79 \mathrm{~ms}$, flip angle $180^{\circ}$, number of slices 40). DTI data were acquired in 30 directions using a single-shot echo planar imaging sequence with a total of 31 volumes of 60 axial slices $\left(b\right.$ value $1=0 \mathrm{~s} / \mathrm{mm}^{2}$ and b value $2=$ $1,000 \mathrm{~s} / \mathrm{mm}^{2}$ ), a slice thickness of $2 \mathrm{~mm}$, TR/TE of 7,800/95 ms, FoV of $256 \times 256 \mathrm{~mm}^{2}$ and an acquisition matrix of $128 \times 128$, resulting in a resolution of $2 \times 2 \times 2 \mathrm{~mm}^{3}$. 

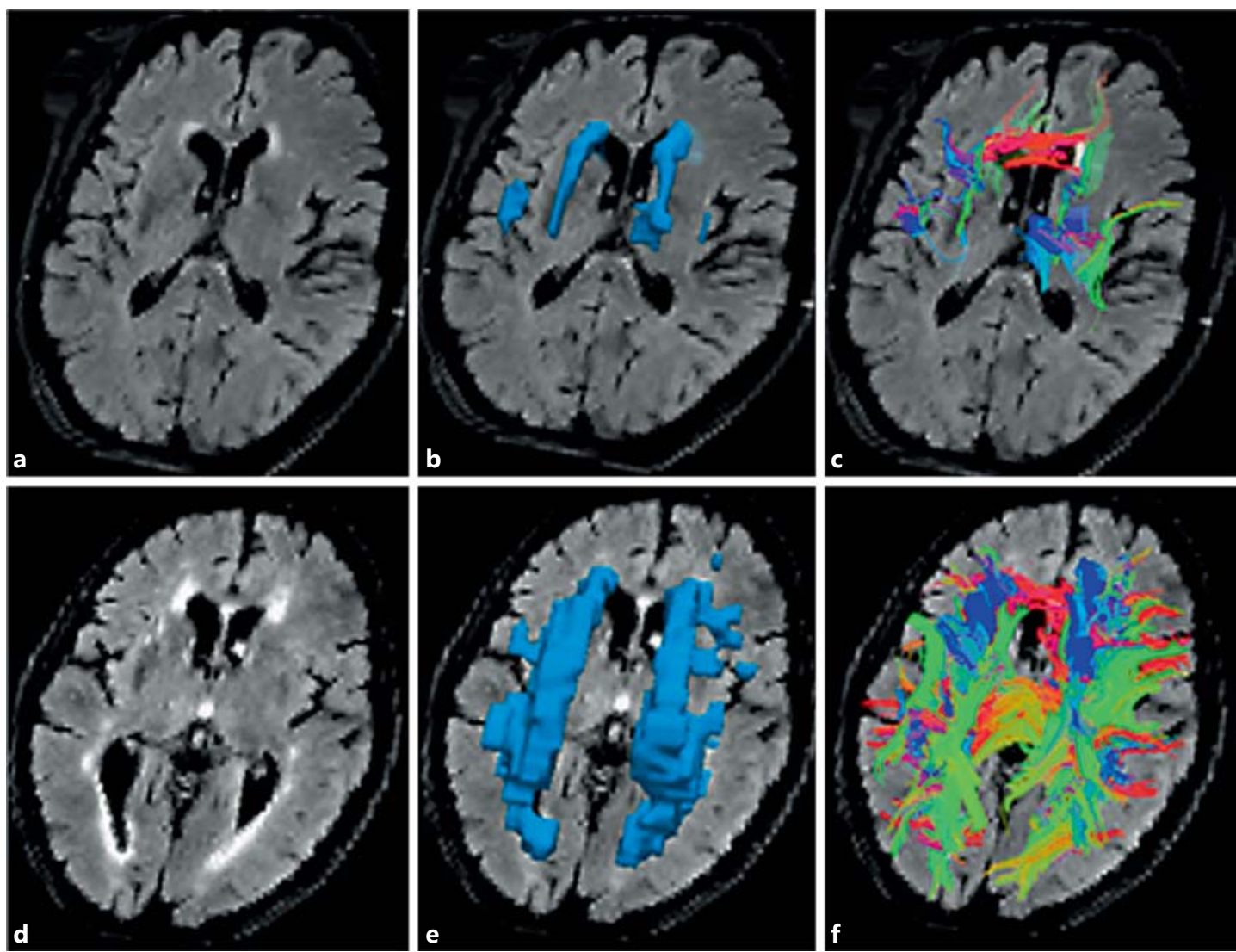

Fig. 1. Quantitative tractography of fibers crossing WMH with small and large volumes of WMH. Low-volume WMH (a-c) and high-volume WMH (d-f). a, d T2 FLAIR MRI with WMH. b, e Three-dimensional region of interest of WMH. c, f Tracts crossing WMH.

\section{Image Analysis}

Image analysis was completed blind to participants' data. Diffusion-weighted images were corrected for eddy current distortions using FMRIB's Diffusion Toolbox in FMRIB Software Library (www.fmrib.ox.ac.uk/fsl/ [33]). DTI reconstruction of the preprocessed data from the FMRIB Software Library was completed with Diffusion Toolkit 0.5 (Ruopeng Wang and Van J. Wedeen at Martinos Center for Biomedical Imaging, Massachusetts General Hospital, Charlestown, Mass., USA; www.trackvis.org). The output included diffusion tensor data and parametric diffusion-weighted image, FA and MD maps. Tracts were assigned in Diffusion Toolkit by the FACT method [34]. Since all tracts were generated for each participant, there was a unique set of fibers generated for each participant. The subject's T2 FLAIR MRI was registered to the diffusion-weighted image map with Slicer 3D 4.1 (www.slicer.org). Tractography data and co-registered T2 FLAIR were analyzed with TrackVis (www.trackvis. org). Although there is high intra-rater and inter-rater variability, expert manual segmentation of WMH is considered the gold standard for measuring WMH in studies of WMH segmentation [35]. All WMH lesions on axial T2 FLAIR were manually segmented (W.R.). The boundaries of individual WMH were clarified with windowing of axial T2 FLAIR slices. Individual WMH lesions were outlined slice-by-slice on all forty axial T2 FLAIR slices for each patient with a mouse-controlled interface on TrackVis. The software generated a single region of interest that included all voxels within outlined areas. The WMH lesion volume was 
Tractography of White Matter Hyperintensities in Elderly Persons

Table 2. Regression coefficients of correlation between WMH tract MD and cognitive test scores in participants with normal cognition or dementia after controlling for age and WMH area

\begin{tabular}{|c|c|c|c|c|c|c|}
\hline & \multicolumn{2}{|c|}{ Total (also controlling for dementia) } & \multicolumn{2}{|c|}{ Cognitively normal } & \multicolumn{2}{|c|}{ Alzheimer's dementia } \\
\hline & $\beta$ & $\mathrm{p}$ value & $\beta$ & $\mathrm{p}$ value & $\beta$ & $\mathrm{p}$ value \\
\hline MMSE & $3,392.75$ & 0.56 & $-3,882.10$ & 0.66 & $14,306.75$ & 0.18 \\
\hline Longest Span Backward & $-7,431.04$ & 0.23 & $-7,982.06$ & 0.31 & $-3,999.74$ & 0.79 \\
\hline Longest Span Forward & $-12,427.88$ & 0.02 & $-14,761.50$ & 0.06 & $-16,742.57$ & 0.14 \\
\hline Stroop test & $-7,195.74$ & 0.91 & $21,840.14$ & 0.80 & $-143,643.13$ & 0.30 \\
\hline Letter Number Sequencing & $-2,649.28$ & 0.81 & $10,661.24$ & 0.48 & $-19,236.97$ & 0.44 \\
\hline
\end{tabular}

$\beta$ represents the nonstandardized regression coefficient. Bold values indicate statistical significance.

measured as the total number of voxels in the whole brain that were within WMH. WMH volume and mean FA and MD for the WMH tracts were quantified with TrackVis. Examples of the quantitative analysis of the MRI scans are shown in figure 1.

\section{Statistical Analysis}

All statistical analyses were conducted using StatPlus. For the overall cohort, including patients with and without dementia, we assessed and reported unstandardized regression coefficients for the relationship between cognitive test scores with WMH tract FA and MD using multiple linear regression adjusting for the effects of age, dementia diagnosis and WMH volume. For participants in each group (normal controls or Alzheimer's dementia), we assessed and reported unstandardized regression coefficients for the relationship between cognitive test scores with WMH tract MD using multiple linear regression adjusting for the effects of age and WMH volume. For continuous variables, comparisons between participants with normal cognition and dementia were made using Student's t test. p values $<0.05$ were considered statistically significant.

\section{Results}

The demographic characteristics of normal participants and patients with dementia are described in table 1 . The average age of the cohort was $73.8 \pm 8.3$ years with an average MMSE score of $26.9 \pm 2.8$. There were no statistically significant age differences between patients with dementia and cognitively normal participants $(\mathrm{p}=0.34)$. Among the participants with normal cognition, there was a greater proportion of female participants than male. There was an approximately equal proportion of male and female participants with dementia. There was a greater WMH area in patients with dementia, but it was not statistically significant (table 1; $\mathrm{p}=0.26$ ). Among the participants with dementia, 4 had hypertension, 2 had well-controlled atrial fibrillation, none had a stroke and only 1 had a lacunar infarct (internal capsule).

In the overall cohort of participants including persons with and without dementia, after controlling for age, dementia diagnosis and WMH volume, there was a statistically significant association between the Longest Span Forward test and MD of WMH tracts $(\beta:-12,428,95 \%$ CI: $-22,678$ to $-2,178, p=0.02$ ). There were no associations between the MD of WMH tracts and scores in the other cognitive tests (table 2). For participants within each group (normal controls or Alzheimer's dementia), there was no association between the MD of WMH tracts and scores in any of the cognitive tests (table 2). 
Reginold et al.: Cognitive Function and 3-Tesla Magnetic Resonance Imaging

Tractography of White Matter Hyperintensities in Elderly Persons

There was no association between the Longest Span Forward test and FA of WMH tracts $(\beta: 4.87, p=0.62)$. There was also no association between the FA of WMH tracts and the MMSE $(\beta: 11.54, p=0.26)$, Longest Span Backward $(\beta:-9.56, p=0.38)$, Stroop test $(\beta:-54.71, p=$ $0.63)$ or Letter Number Sequencing $(\beta:-7.61, p=0.69)$.

\section{Discussion}

A novel correlation was detected between the MD of WMH tracts and performance on the Wechsler Memory Scale-III Longest Span Forward. Earlier tractography studies of WMH using the TBSS procedure [26-28] detected associations between the MD/FA of common tracts and the MMSE [28], Trails, Stroop test [26], Wisconsin Card Sorting, Longest Span Forward and Longest Span Backward [27]. These white matter tracts may or may not have crossed WMH in each subject. In this study, we overcame this limitation by using deterministic tractography with the FACT procedure to create unique fiber tracts for each subject. By measuring only tracts that crossed WMH in each subject, we found fewer associations with cognitive tests than earlier studies. Our results provided more evidence that cognitive dysfunction could be due to 'disconnection' of tracts by WMH, as a greater MD of WMH tracts was associated with worse performance on the Longest Span Forward test.

Other DTI studies of WMH focused measurements within WMH lesions. Two of the five studies measuring FA/MD within WMH detected associations with performance on cognitive testing $[7,8]$. The MD of WMH was associated with cognitive tests including the Rey Recall of Words, Stroop test, Letter Digit Subtraction and Digit Cancelling. An earlier study that included the Longest Span Forward was unable to demonstrate an association with WMH FA/MD [10]. The novel association between this test and WMH in our study may be related to the use of tractography. Tracts crossing WMH incorporated the fiber tract proximal and distal to the WMH lesions. Although these proximal and distal tract segments appeared normal on T2 FLAIR, there may have been microstructural injury detectable only by DTI. In tractography studies of lacunes and subcortical infarcts, degeneration of the fiber tract was detected proximal and distal to the visible lesion [36-39]. DTI studies of WMH have detected injury in the normal-appearing white matter (NAWM) adjacent to WMH lesions [40]. Histologically, adjacent NAWM contains looser axonal networks and reduced myelin density [1]. Earlier studies have correlated the injury in NAWM with cognitive function [9-11]. Compared to measures within WMH, measuring WMH tracts may incorporate additional white matter injury remote from the WMH lesions. Future studies could assess if there is injury in the fiber tract proximal and distal to the WMH lesions.

There may have been limitations in this study related to sample size and technique. We may not have detected a correlation between tract MD and performance on Longest Span Forward in the normal control and Alzheimer's dementia subgroups due to their smaller sample sizes and limited range in cognitive function compared to the overall cohort. Tractography relies on anisotropy to track fibers [31]. The reduction in anisotropy at WMH lesions could have caused tracks to incorrectly terminate or deviate at the lesion [41, 42]. We may not have detected an association between cognitive function and FA of WMH tracts due to the dependence on anisotropy for tracking [13]. Consistent with earlier DTI studies of WMH, our measurements of MD and FA were averaged over all lesions irrespective of their location [7, 9-11]. It may have been advantageous to measure diffusion characteristics of specific fiber tracts. Given the variable location of WMH lesions in subjects and our small sample size, this study would be underpowered for analysis of the effects of WMH in specific fiber tracts.

Despite these limitations, our study demonstrated the potential for DTI tractography to predict performance on cognitive testing in persons with WMH. In the elderly population 
Reginold et al.: Cognitive Function and 3-Tesla Magnetic Resonance Imaging

Tractography of White Matter Hyperintensities in Elderly Persons

with a high prevalence of WMH, DTI tractography could become helpful in triaging patients for further cognitive testing. Future studies with larger sample sizes should assess the impact of WMH in strategic locations and particular fiber tracts and determine the screening and diagnostic cutoffs of WMH tract MD for cognitive dysfunction in elderly patients. As advances are made in tractography, the MD of WMH tracts may be an important tool in providing clinicians with insight into the significance of incidental WMH lesions for patients.

\section{Statement of Ethics}

All procedures followed were in accordance with the ethical standards of the responsible committee on human experimentation (institutional and national) and with the Helsinki Declaration of 1975, as revised in 2000. Informed consent was obtained from all patients for being included in the study.

\section{Disclosure Statement}

The authors declare that they have no conflicts of interest.

\section{References}

1 Gouw AA, Seewann A, van der Flier WM, et al: Heterogeneity of small vessel disease: a systematic review of MRI and histopathology correlations. J Neurol Neurosurg Psychiatry 2011;82:126-135.

-2 Ylikosi A, Erkinjuntti T, Rainiko R, et al: White matter hyperintensities on MRI in the neurologically nondiseased elderly. Analysis of cohorts of consecutive subjects aged 55 to 85 years living at home. Stroke 1995;26: 1171-1177.

3 Debette S, Markus HS: The clinical importance of white matter hyperintensities on brain magnetic resonance imaging: systematic review and meta-analysis. BMJ 2010;26:c3666.

4 Pantoni L, Poggesi A, Inzitari D: The relation between white-matter lesions and cognition. Curr Opin Neurol 2007;20:390-397.

5 Patel B, Markus HS: Magnetic resonance imaging in cerebral small vessel disease and its use as a surrogate disease marker. Int J Stroke 2011;6:47-59.

6 Taylor WD, Payne ME, Krishnan KR, et al: Evidence of white matter tract disruption in MRI hyperintensities. Biol Psychiatry 2001;50:179-183.

7 Vernooij MW, Ikram MA, Vrooman HA, et al: White matter microstructural integrity and cognitive function in a general elderly population. Arch Gen Psychiatry 2009;66:545-553.

-8 Viana-Baptista M, Bugalho P, Jordão C, et al: Cognitive function correlates with frontal white matter apparent diffusion coefficients in patients with leukoaraiosis. J Neurol 2008;255:360-366.

-9 Lee DY, Fletcher E, Martinez 0, et al: Regional pattern of white matter microstructural changes in normal aging, MCI, and AD. Neurology 2009;73:1722-1728.

10 Schmidt R, Ropele S, Ferro J, et al: Diffusion-weighted imaging and cognition in the leukoaraiosis and disability in the elderly study. Stroke 2010;41:e402-e408.

11 O'Sullivan M, Morris RG, Huckstep B, et al: Diffusion tensor MRI correlates with executive dysfunction in patients with ischaemic leukoaraiosis. J Neurol Neurosurg Psychiatry 2004;75:441-447.

$\checkmark 12$ O’Sullivan M, Jones DK, Summers PE, et al: Evidence for cortical 'disconnection' as a mechanism of age-related cognitive decline. Neurology 2001;57:632-638.

$\checkmark 13$ Johansen-Berg H, Behrens TEJ: Just pretty pictures? What diffusion tractography can add in clinical neuroscience. Curr Opin Neurol 2006;19:379-385.

14 Davis SW, Dennis NA, Buchler NG, et al: Assessing the effects of age on long white matter tracts using diffusion tensor tractography. Neuroimage 2009;46:530-541.

15 Zahr NM, Rohlfing T, Pfefferbaum A, et al: Problem solving, working memory, and motor correlates of association and commissural fiber bundles in normal aging: a quantitative fiber tracking study. Neuroimage 2009; 44:1050-1062.

16 Voineskos AN, Rajji TK, Lobaugh NJ, et al: Age-related decline in white matter tract integrity and cognitive performance: a DTI tractography and structural equation modeling study. Neurobiol Aging 2012;33:21-34.

17 Penke L, Maniega SM, Bastin ME, et al: Brain white matter tract integrity as a neural foundation for general intelligence. Mol Psychiatry 2012;17:1026-1030. 
18 Sasson E, Doniger GM, Pasternak 0, et al: White matter correlates of cognitive domains in normal aging with diffusion tensor imaging. Front Neurosci 2013;7:32.

19 Booth T, Bastin ME, Penke L, et al: Brain white matter tract integrity and cognitive abilities in communitydwelling older people: the Lothian Birth Cohort, 1936. Neuropsychology 2013;27:595-607.

20 Gao J, Cheung RT, Chan YS, et al: The relevance of short-range fibers to cognitive efficiency and brain activation in aging and dementia. PLoS One 2014;9:e90307.

21 Nakata Y, Sato N, Abe 0, et al: Diffusion abnormality in posterior cingulate fiber tracts in Alzheimer's disease: tract-specific analysis. Radiat Med 2008;226:466-473.

22 Morikawa M, Kiuchi K, Taoka T, et al: Uncinate fasciculus-correlated cognition in Alzheimer's disease: a diffusion tensor imaging study by tractography. Psychogeriatrics 2010;10:15-20.

23 Taoka T, Morikawa M, Akashi T, et al: Fractional anisotropy - threshold dependence in tract-based diffusion tensor analysis: evaluation of the uncinate fasciculus in Alzheimer disease. AJNR Am J Neuroradiol 2009;30: 1700-1703.

24 Pievani M, Agosta F, Pagani E, et al: Assessment of white matter tract damage in mild cognitive impairment and Alzheimer's disease. Hum Brain Mapp 2010;31:1862-1875.

25 Huston JM, Field AS: Clinical applications of diffusion tensor imaging. Magn Reson Imaging Clin N Am 2013; 21:279-298.

26 Quinque EM, Arélin K, Dukart J, et al: Identifying the neural correlates of executive functions in early cerebral microangiopathy: a combined VBM and DTI study. J Cereb Blood Flow Metab 2012;32:1869-1878.

27 Soriano-Raya JJ, Miralbell J, López-Cancio E, et al: Tract-specific fractional anisotropy predicts cognitive outcome in a community sample of middle-aged participants with white matter lesions. J Cereb Blood Flow Metab 2014;34:861-869.

28 Otsuka Y, Yamauchi H, Sawamoto N, et al: Diffuse tract damage in the hemispheric deep white matter may correlate with global cognitive impairment and callosal atrophy in patients with extensive leukoaraiosis. AJNR Am J Neuroradiol 2012;33:726-732.

29 Folstein MF, Folstein SE, McHugh PR: 'Mini-mental state'. A practical method for grading the cognitive state of patients for the clinician. J Psychiatr Res 1975;12:189-198.

30 Wechsler D: Wechsler Memory Scale, ed 3. San Antonio, TX, The Psychological Corporation, 1997.

31 Stroop JR: Studies of interference in serial verbal reactions. J Exp Psychol 1935;18:643-662.

-32 McKhann G, Drachmna D, Folstein M, et al: Clinical diagnosis of Alzheimer's disease: report of the NINCDSADRDA Work Group under the auspices of Department of Health and Human Services Task Force on Alzheimer's Disease. Neurology 1984;34:939-944.

33 Smith SM, Jenkinson M, Woolrich MW, et al: Advances in functional and structural MR image analysis and implementation as FSL. Neuroimage 2010;23:S208-S219.

34 Wiegell MR, Larsson HB, Wedeen VJ, et al: Fiber crossing in human brain depicted with diffusion tensor MR imaging. Radiology 2000;217:897-903.

-35 Simoes R, Mönninghoff C, Dlugaj M, et al: Automatic segmentation of cerebral white matter hyperintensities using only 3D FLAIR images. Magn Reson Imaging 2013;31:1182-1189.

-36 Kloppenborg RP, Nederkoorn PJ, Geerlings MI, et al: Presence and progression of white matter hyperintensities and cognition: a meta-analysis. Neurology 2014;82:2127-2138.

-37 Mukherjee P: Diffusion tensor imaging and fiber tractography in acute stroke. Neuroimaging Clin N Am 2005; 15:655-665.

-38 Liang Z, Zeng J, Liu S, et al: A prospective study of secondary degeneration following subcortical infarction using diffusion tensor imaging. J Neurol Neurosurg Psychiatry 2007;78:581-586.

-39 Radlinska B, Ghinani S, Leppert IR, et al: Diffusion tensor imaging, permanent pyramidal tract damage, and outcome in subcortical stroke. Neurology 2010;75:1048-1054.

40 Reijmer YD, Freeze WM, Leemans A, et al: The effect of lacunar infarcts on white matter tract integrity. Stroke 2013;44:2019-2021.

41 Maillard P, Fletcher E, Harvey D, et al: White matter hyperintensity penumbra. Stroke 2011;42:1917-1922.

42 Hua K, Zhang J, Wakana S, et al: Tract probability maps in stereotaxic spaces: analyses of white matter anatomy and tract-specific quantification. Neuroimage 2008;39:336-347. 